\title{
How to Predict SARS-COV2 Infection in Patient Admitted in Emergency Department: A Prospective Cohort Study.
}

Frederic BALEN ( $\square$ fred.balen@gmail.com )

CHU Toulouse: Centre Hospitalier Universitaire de Toulouse https://orcid.org/0000-0002-4340-7391

\section{Sandrine CHAPENTIER}

CHU Toulouse

Paul-Henri AUBOIROUX

CHU Toulouse

Elise NOEL-SAVINA

CHU Toulouse

Nicolas SANS

CHU Toulouse

Gaspard GROUTEAU

CHU Toulouse

Anne RAYNAUD

CHU Toulouse

Manon HEBRARD

CHU Toulouse

Xavier DUBUCS

CHU Toulouse

Charles-Henri HOUZE-CERFON

CHU Toulouse

Hélène COLINEAUX

CHU Toulouse

Original research

Keywords: COVID19, Prediction model, Emergency Department

Posted Date: December 9th, 2020

DOI: https://doi.org/10.21203/rs.3.rs-122151/v1 
License: (c) (i) This work is licensed under a Creative Commons Attribution 4.0 International License. Read Full License 


\section{Abstract}

Background. In order to rapidly identifying patients with a low probability of being infected by COVID19 to quickly refer them to specialized departments, the objective of our study was to develop a clinical predictive model of infection by COVID19 in patients attending the ED for respiratory symptoms or unexplained fever.

Methods. We included all patients over 15 years old, admitted in one of the 2 emergency departments of Toulouse University Hospital between March 13 and March 31 for respiratory symptoms (dyspnea, cough), or fever (or sensation of fever) of unknown origin, and potentially requiring hospitalization. COVID19 infection was assessed by CT-SCAN and RT-PCR. All the candidate predictors were variables collected during the first clinical examination. Internal validation of the final model was performed using the bootstrap procedure. We performed a temporal validation in the same way on patients included between April 1 and April 13.

Results. 772 patients were included. The prevalence of COVID19 was $25.5 \%$. There were 19 predictors in the final model. The corrected-by-optimism area under the curve was $0.86(95 \% \mathrm{Cl}=[0.83 ; 0.89])$. For a threshold at $10 \%$, the sensitivity was $92 \%$, the specificity was $56 \%$, and the false negative rate was $5 \%$. In secondary data, including 387 patients, the prevalence of COVID19 was $15 \%$. The area under the curve was $0.73(95 \% \mathrm{Cl}=[0.63 ; 0.83])$. For the same threshold, the sensitivity was $78 \%$, the specificity was $48 \%$, and the false negative rate was $7 \%$

Conclusion. We have developed a predictive tool of COVID19 infection for patients attending the ED. It could safely reduce admission in COVID19 dedicated unit in ED and prevent its overcrowding.

Trial registration number: NCT: RC31/20/0149

\section{Background}

Infection with coronavirus SARS-COV2 (COVID19) is a pandemic disease that started in China during the last month of 2019 and spread to Europe in the beginning of 2020 (1). The severity of this viral infection is mainly due to a respiratory syndrome $(2,3)$.

During these times of pandemic, Emergency Departments (ED) have had to change their organization (4, 5). There are two challenges for the Emergency Physicians facing the COVID19 pandemic: first, rapidly assessing the risk of patients admitted for respiratory symptoms to be infected by COVID19, in order to avoid contamination to other patients and medical teams (6); and second, paying particular attention to patients at risk of severe lung injury, to anticipate their admission in Intensive Care Unit (ICU) for mechanical ventilation.

Since the beginning of the epidemic, many predictive models have been developed, including prognostic models assessing the risk of being hospitalized, the risk of developing a severe form, or the risk of dying 
(7-11). Numerous models have also been developed to predict COVID19 among patients with respiratory symptom or unexplained fever(6). However, those models were requiring CT-scan or blood test results. Therefore most of these studies have been criticized for their lack of methodological rigor, transparency and precision concerning their potential usefulness (11).

We are looking at a quick identification (i.e. before the results of imaging and biological sampling including nasopharyngeal sampling) among patients presenting respiratory symptoms or unexplained fever, those with a low probability of being infected by COVID19. We wanted to identify these patients in order to be able to quickly refer them to non-COVID ED unit without increasing the risk of infecting other patients or care providers in these units. This could also save time for acute patients and resource consumption for the ED.

The objective of our study was therefore to develop a clinical predictive model of infection by COVID19 in patients attending the ED for respiratory symptoms or unexplained fever and which require hospitalization. The variables used in this model have to be available at triage.

\section{Methods}

\section{Study design and setting}

This prospective observational pragmatic cohort study took place during the COVID19 epidemic between March 13 and April 13, 2020 in the two EDs of an University Hospital. Those two EDs are located in north and south of our city. The EDs treated 78,000 and 40,000 visitors in 2018 , respectively. During the pandemic, these EDs were re-organized to propose specific units for suspected COVID19 patients in order to segregate COVID19 activity from standard activity. Patients suspected of COVID19 were patients with dyspnea or unexplained fever. The medical dispatch center systematically addressed suspected COVID19 patients to these dedicated units.

A CT-scan was dedicated in each ED to this activity and these units were the only centers of the area to provide COVID19 testing by Reverse Transcriptase - Polymerase Chain Reaction (RT-PCR) daily. After admission in one of these units, patients underwent a chest CT-scan if the emergency practitioner estimated that they would potentially require hospitalization. Patients were tested by COVID19 RT-PCR (nasopharyngeal swab or throat swab) if the chest CT-scan could not exclude COVID19 infection. This strategy was justified by the lack of sensitivity of RT-PCR and the poor specificity of CT-scans (12).

\section{Source of data}

Standardized observations were completed for all patients admitted between March 13 and March 31 in one of the COVID19 dedicated units during the COVID19 epidemic in the two EDs of the University Hospital of Toulouse, France. These are the data used for the development and internal validation of the model (original data). Data were collected in the same way between April 1 and April 13 for the temporal validation (secondary data). 


\section{Participants}

Inclusion criteria were: patients over 15 years old, admitted in ED for respiratory symptoms (dyspnea, cough) or fever (or sensation of fever) of unknown origin and potentially requiring hospitalization. We decided that the patient was considered by the physician who performed the first clinical examination, as potentially in need of complementary tests or hospitalization, if he or she performed a chest CT-scan, because this was the care protocol implemented within the COVID19 dedicated units. We excluded patients with already confirmed COVID19 infection on admission to the emergency department, as these patients are not part of the model's target population. The inclusion and exclusion criteria were the same for original and secondary data.

\section{Measures}

Candidate predictors were demographic characteristics (age, sex, autonomy), medical history (asthma, cardio-respiratory disease, obesity, cancer, treatment), disease history (duration, symptoms), and clinical examination (signs of heart failure, pulmonary auscultation, digestive signs, etc.). These variables were collected by the physicians during the first clinical examination.

The outcome was the diagnosis of COVID19 infection. Final diagnosis of COVID19 was assessed as follows (Fig. 1): in case of negative chest CT-scan, patient was considered as COVID19 negative ; in case of positive CT-scan and positive RT-PCR, patient was considered as COVID19 positive; in case of CT-scan results "not typical" of COVID19 infection and negative RT-PCR, patient was considered as COVID19 negative; in case of a very suspect COVID19 lesion of at the CT-scan and negative RT-PCR, patient was tested for other respiratory virus and was reassessed by a second expert physician (respiratory or infectious disease specialist).

\section{Sample size}

During the implementation of the study, we had assumed an average prevalence of $15 \%$ of COVID 19 positivity among the eligible population over the inclusion period (first phase of the virus diffusion at the regional level), about 10 candidate-predictors and a minimum $\mathrm{R}^{2}$ of 0.15 . We estimated the number of events (COVID19 positive patients) required at a minimum of 83 (13), i.e. a total number of subjects required of 553 patients.

\section{Statistical analysis: development}

Analysis were performed with $\mathrm{R}$ release 3.6.1(14) and $\mathrm{R}$ studio release 1.2.5001.

We performed a complete-case analysis. No imputation was performed. One important variable ("Anosmia or ageusia") had more than $50 \%$ of missing data because it was not collected from the beginning, so we used the class "not known" in order to keep both the variable and the patients with missing data on the model. 
We compared several modeling and screening approaches by cross-validation using variables associated with outcome with a p-value $<0.20$ in bivariate analysis. Logistic regression with stepwise selection have been selected as better approach, based on optimization of C-statistic. We verified stability of the selected variables ("bootStepAIC" package) and stability of the coefficient estimation by bootstrap (100 and 200 replications respectively).

\section{Statistical analysis: internal validation}

The model has been developed on the entirety of original data. Performance, calibration and discrimination parameters have also been estimated on the total base ("apparent parameters") then corrected by optimism ("corrected parameters"). The optimism has been estimated by bootstrap (15).

We especially estimated the corrected $\mathrm{R}^{2}$, the corrected intercept and slope of calibration equation and the bias-corrected Somers D with the "rms" package developed by Harrell (16). We estimated the corrected AUROC (C-statistic) with the same approach. Then, apparent and corrected-by-optimism sensitivities, specificities, accuracies, error rates and predictive values were estimated for several thresholds. As we wanted to minimize the risk of infecting non-COVID19 units, the threshold was chosen with a view of limiting the number of false negatives.

\section{Statistical analysis: temporal validation}

Based on the final model developed in the original data, we estimated AUROC (C-statistic) and sensitivities, specificities, accuracies, error rates and predictive values for several thresholds on patients of the secondary data.

\section{Standardized redaction}

In order to improve our methodology, we used the TRIPOD checklist to report our methods and results

\section{Ethical approval}

The study protocol was approved by the Ethics Committee Ouest-6 France (Brest: 1276 HPS 3) (NCT: RC31/20/0149). Patients received information on their participation at the study. No signed consent was required.

\section{Results}

\section{Description of original population}

During the 19-day inclusion period, 772 patients were admitted to the emergency department (ED) with respiratory signs or unexplained fever and were considered at admission as potentially in need of hospitalization (see flow chart on Supplementary data). The median age was 58 and $58.5 \%$ were women (see Table 1). The prevalence of COVID19 in this population was $25.5 \%$. 


\section{Factors associated with COVID19 infection}

In the first screening, twenty-eight variables were associated with COVID19. After logistic regression, only 19 clinical variables remained associated in multivariable analysis. The coefficient estimations of the final model are given in Table 2. Factors associated with COVID19 infection are: older age, male sex, preserved autonomy, obesity, diabetes, normal systolic blood pressure, hyperthermia, anosmia, duration of symptoms form 5 to 13 days, hypoxemia (i.e. SpO2 <95\%), high respiratory frequency, and cracklings at the clinical examination. Meanwhile, variables associated with absence of COVID19 infection are: brutal start of dyspnea, history of COPD, thoracic pain, nausea, tachycardia, and ronchii or edema of low limb at clinical examination.

\section{Predictive model}

Our predictive model had a performance estimated by corrected area under the curve of $0.86(95 \% \mathrm{Cl}=$ $[0.83 ; 0.89])$. The performances according to the threshold are given in Table 3. Considering a threshold of $10 \%$ permitted to class $43 \%$ of the population in a "non-COVID" pathway with a negative predictive value of $95 \%$.

\section{Temporal validation}

During the 13-day inclusion period for secondary data, 387 patients were admitted to the emergency department with respiratory signs or unexplained fever and were considered at admission as potentially in need of hospitalization (see flow chart in Supplementary data). The median age was 59 and $53.2 \%$ were women (see Table in supplementary data). The prevalence of COVID19 in this population was 15\%.

In these secondary data, the area under the curve was $0.73(95 \% \mathrm{Cl}=[0.63 ; 0.83])$. Sensitivity also appears to be lower. Although the false negative rate remains low, the rate of false positives increases sharply. This could be partially explained by the lower prevalence of COVID-positive patients in this validation cohort.

\section{Discussion}

\section{Main result}

We found simple clinical factors associated with COVID19 infection in patients admitted in ED with dyspnea during COVID19 pandemic. Those factors can be assessed at first medical contact and can be used at triage to refer patient in COVID19 or in non-COVID19 ED unit. While it remains important to propose dedicated COVID19 unit in ED to avoid infection of non-COVID patients and ED staff (6), our predicting model classified nearly $50 \%$ of patients in a "standard pathway", with a false negative rate of 5 to $7 \%$. This could save time for acute patients and resource consumption for the ED. In the actual context 
of persistent admission in ED for suspected COVID19 patients, provide a simple strategy of triage of those patients remain important. It requires a simple informatics program to be used by a triage nurse (our tool is available at https://tlsepredcovid19.shinyapps.io/app-1/, for example).

However, a false negative rate above $5 \%$ could be consider unacceptable. To improve those performance, we could imagine integrating point-of-care ultrasound (17-20), but that may lead to longer duration of triage.

\section{Comparison with the literature}

The higher rate of COVID19 patients in the first derivation cohort (25.5\%) compared to the validation cohort (15\%) is explained by the temporal repartition of cases. The COVID19 rate in studies about COVID19 diagnosis is extremely variable, due to differences in inclusion criteria and epidemic severity in the area of study.

The predictors of COVID19 infection in our model are globally consistent with the literature (11): age, male sex, fever, anosmia (21), shortness of breath and duration of symptoms from 5 to 13 days (2). Institutionalized patients are unlikely to be COVID19 positive, probably due to the protection measures that were quickly proposed in France for those patients. Meanwhile non-institutionalized "frail" patients (i.e. diabetics and patients requiring diuretics as their usual treatment) seem more likely to be COVID19 positive and to require ED visits. Sudden onset and abnormal hemodynamic parameters are associated with alternative diagnoses of dyspnea. Gastrointestinal symptoms are associated with COVID19 negative patients in our cohort, while this sign was supposed to be frequent in COVID19 patients $(7,8)$. However, few studies about diagnosis found gastrointestinal symptoms to be associated with COVID19 in multivariate analysis (11).

\section{Strengths and limits}

Prospective inclusion of patients with standardized collection of variable leads to few missing values (except for the anosmia variable that was introduced after the start of the study, which induced an important rate of missing values). In order to improve our methodology, we used the TRIPOD checklist to report our methods and results. We tried to be transparent and precise on their desired usefulness for the model, and we evaluated their performances consistently for this intended utility.

The principal limit of our work is its monocentric character. We cannot therefore exclude possible specificities of this French population, which limits the generalizability of the results. This also led to an absence of external validation that requires further studies. We are open to collaboration to carry out this external validation on other data or to make our data available.

\section{Conclusion}


We have developed a predictive tool of COVID19 infection for patients attending the ED. It could safely reduce admission in COVID19 dedicated unit in ED and prevent its overcrowding.

\section{Declarations}

Ethics approval and consent to participate

The study protocol was approved by the Ethics Committee Ouest-6 France (Brest: 1276 HPS 3) (NCT: RC31/20/0149). Patients received information on their participation at the study. No signed consent was required.

Consent for publication: Not applicable.

Availability of data and materials

The datasets used and/or analysed during the current study are available from the corresponding author on reasonable request.

Competing interest: The authors declare that they have no competing interests.

Funding: None.

Authors' contribution:

FB designed the study and drafted the manuscript.

SC, PHA, ENS, NS, GG, AR, XD, CHHC provide critical revision of the manuscript for important intellectual content.

MH performed acquisition of the data.

$\mathrm{HC}$ analyzed and interpreted the data and drafted the manuscript.

Acknowledgment

The authors thank Dr MOULIS Guillaume for his critical review of the manuscript.

\section{References}

1. COVID-19 situation reports [Internet]. [cited 2020 May 9]. Available from: https://www.who.int/emergencies/diseases/novel-coronavirus-2019/situation-reports/

2. Guan W-J, Ni Z-Y, Hu Y, Liang W-H, Ou C-Q, He J-X, et al. Clinical characteristics of coronavirus disease 2019 in China. N Engl J Med. 2020 Feb 28; 
3. Grasselli G, Zangrillo A, Zanella A, Antonelli M, Cabrini L, Castelli A, et al. Baseline Characteristics and Outcomes of 1591 Patients Infected With SARS-CoV-2 Admitted to ICUs of the Lombardy Region, Italy. JAMA. 2020 Apr 6;

4. Lu K-L, Chen S, Leung L-P. Initial experience of an emergency department in shenzhen in responding to the emerging wuhan coronavirus pneumonia. Ann Emerg Med. 2020;75(4):556.

5. Cao Y, Li Q, Chen J, Guo X, Miao C, Yang H, et al. Hospital Emergency Management Plan During the COVID-19 Epidemic. Acad Emerg Med. 2020;27(4):309-311.

6. Wee LE, Fua T-P, Chua YY, Ho FWA, Sim XYJ, Conceicao EP, et al. Containing COVID-19 in the emergency room: the role of improved case detection and segregation of suspect cases. Acad Emerg Med. 2020 Apr 12;

7. Huang C, Wang Y, Li X, Ren L, Zhao J, Hu Y, et al. Clinical features of patients infected with 2019 novel coronavirus in Wuhan, China. Lancet. 2020 Feb 15;395(10223):497-506.

8. Zhou F, Yu T, Du R, Fan G, Liu Y, Liu Z, et al. Clinical course and risk factors for mortality of adult inpatients with COVID-19 in Wuhan, China: a retrospective cohort study. Lancet. 2020 Mar 28;395(10229):1054-1062.

9. Richardson S, Hirsch JS, Narasimhan M, Crawford JM, McGinn T, Davidson KW, et al. Presenting Characteristics, Comorbidities, and Outcomes Among 5700 Patients Hospitalized With COVID-19 in the New York City Area. JAMA. 2020 Apr 22;

10. Hu H, Yao N, Qiu Y. Comparing rapid scoring systems in mortality prediction of critical ill patients with novel coronavirus disease. Acad Emerg Med. 2020 Apr 20;

11. Wynants L, Van Calster B, Collins GS, Riley RD, Heinze G, Schuit E, et al. Prediction models for diagnosis and prognosis of covid-19 infection: systematic review and critical appraisal. BMJ. 2020 Apr 7;369:m1328.

12. Kim H, Hong H, Yoon SH. Diagnostic Performance of CT and Reverse Transcriptase-Polymerase Chain Reaction for Coronavirus Disease 2019: A Meta-Analysis. Radiology. 2020 Apr 17;201343.

13. Riley RD, Ensor J, Snell KIE, Harrell FE, Martin GP, Reitsma JB, et al. Calculating the sample size required for developing a clinical prediction model. BMJ. 2020 Mar 18;368:m441.

14. R: a language and environment for statistical computing [Internet]. [cited 2020 Jul 13]. Available from: https://www.gbif.org/tool/81287/r-a-language-and-environment-for-statistical-computing

15. Steyerberg EW, Harrell FE, Borsboom GJ, Eijkemans MJ, Vergouwe Y, Habbema JD. Internal validation of predictive models: efficiency of some procedures for logistic regression analysis. J Clin Epidemiol. 2001 Aug;54(8):774-781.

16. Harrell FE. Regression Modeling Strategies. New York, NY: Springer New York; 2001.

17. Moore S, Gardiner E. Point of care and intensive care lung ultrasound: A reference guide for practitioners during COVID-19. Radiography (Lond). 2020 Apr 17;

18. Soldati G, Smargiassi A, Inchingolo R, Buonsenso D, Perrone T, Briganti DF, et al. Is There a Role for Lung Ultrasound During the COVID-19 Pandemic? J Ultrasound Med. 2020 Jul;39(7):1459-1462. 
19. Soldati G, Smargiassi A, Inchingolo R, Buonsenso D, Perrone T, Briganti DF, et al. Proposal for International Standardization of the Use of Lung Ultrasound for Patients With COVID-19: A Simple, Quantitative, Reproducible Method. J Ultrasound Med. 2020 Jul;39(7):1413-1419.

20. Peyrony O, Marbeuf-Gueye C, Truong V, Giroud M, Rivière C, Khenissi K, et al. Accuracy of emergency department clinical findings for diagnosis of coronavirus disease 2019. Ann Emerg Med. 2020 May 21 ;

21. Kai Chua AJ, Yun Chan EC, Loh J, Charn TC. Acute olfactory loss is specific for Covid-19 at the Emergency Department. Ann Emerg Med. 2020 May 14;

\section{Figures}

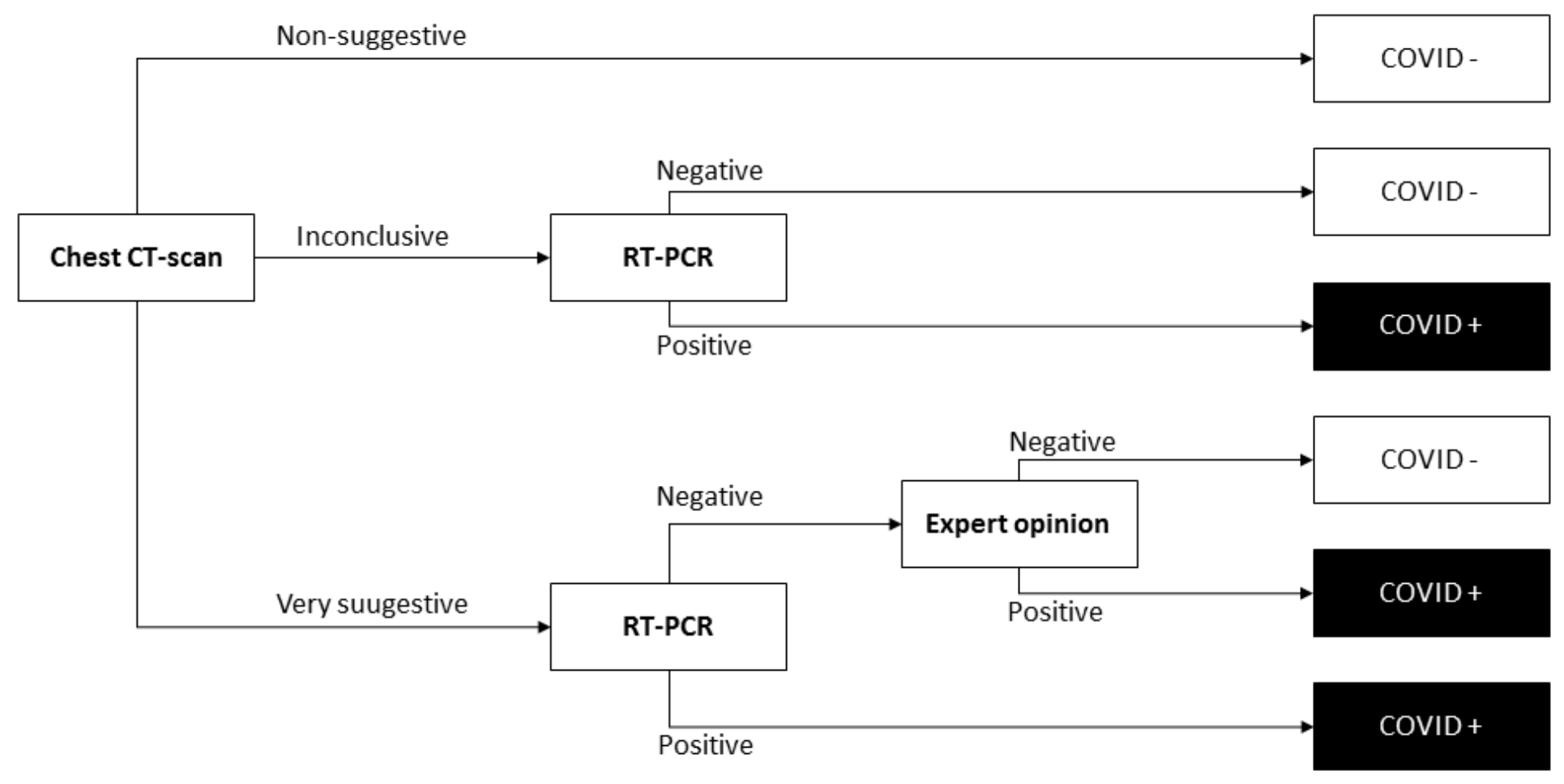

\section{Figure 1}

Assessment of COVID 19 infection

\section{Supplementary Files}

This is a list of supplementary files associated with this preprint. Click to download.

- Table1.xlsx

- SF1.tif

- Table3.xlsx

- Table4.xlsx 
- Table2.xIsx

- SF2.tif

Page 12/12 